\title{
Utilización de la placa dorsal «Pi» en las fracturas articulares graves del extremo distal del radio
}

\author{
J. G. Boretto(1), V. A. Alfie ${ }^{(1)}$, W. O. Bourgeois ${ }^{(1)}$, G. L. Gallucci ${ }^{(2)}$, \\ P. De CARLI ${ }^{(3)}$ \\ ${ }^{(1) B E C A R I O}$ de Especialización de Cirugía de Mano y Miembro Superior. \\ ${ }^{(2)}$ Cirujano de MaNo y Miembro Superior. \\ (3) JeFE DEL SECTOR DE CiRUGía DE MANO Y MIEMBRo SuPERIOR. \\ Hospital ITALIANO DE BUENOS AIRES. \\ InStituto de ORTOPEDia Y TRAUMatología «PROF. CARLos OtTOLENGHI». \\ Sector de Cirugía de Mano y Miembro Superior.
}

Correspondencia:

Dr. Jorge G. Boretto

Instituto de Ortopedia y Traumatología «Prof. Carlos Ottolenghi»

Hospital Italiano de Buenos Aires

Potosí 4215. Capital Federal

C1199ACK Ciudad Autónoma de Buenos Aires, Buenos Aires. Argentina

T.E. 4959-0200 int. 4943/ Fax 4981-0991

E-mail: jboretto@hotmail.com

Se presentan los resultados obtenidos en una serie de 24 fracturas Tipo C3 (AO) del extremo distal del radio, tratadas con la placa «Pi» como único material de osteosíntesis. Se valoró el arco de movilidad, la fuerza de prensión así como parámetros radiográficos. Los pacientes recuperaron el $80 \%$ de flexo-extensión, el $82 \%$ de desviación radio-cubital, el $97 \%$ de prono-supinación y el $87 \%$ de la fuerza de prensión con respecto al lado sano. En la radiología simple, se obtuvo: $4^{\circ}$ de inclinación palmar, $22^{\circ}$ de inclinación radial, $11 \mathrm{~mm}$ en la longitud radial y $0,5 \mathrm{~mm}$ de escalón articular. Según la escala de Gartland y Werley, 16 casos obtuvieron excelentes resultados y 8 buenos. A pesar de que en 9 pacientes se tuvo que extraer el implante, los autores recomiendan su uso en el tratamiento de estas graves lesiones articulares.

Palabras claves: Placa Pi, Fracturas articulares de muñeca, Fracturas $C 3$ (AO) de radio distal.
The results of a series of 24 cases of Type C3 (AO) fractures of the distal radius are presented, treated with a $« \mathrm{Pi} »$ plate as the only fixation device. The range of movement, grip strength and radiographic parameters were measured. Patients recovered $80 \%$ of flexion-extension, $82 \%$ of radio-ulnar deviation, $97 \%$ of prono-supination and $87 \%$ of grip strength compared to the other side. Plain radiographs demonstrated: $4^{\circ}$ of volar inclination, $22^{\circ}$ of radial inclination, $11 \mathrm{~mm}$ of radial length and $0.5 \mathrm{~mm}$ of intra-articular step. According to the scoring system by Gartland and Werley, 16 cases obtained and excellent result and 8 were good. In spite of the fact that 9 cases required removal of the implant, the authors recommend its use in the treatment of these severe intra-articular injuries.

Key words: Pi plate, intra-articular wrist fractures, Fractures C3 (AO) of distal radius. 


\section{INTRODUCCION}

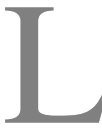

as fracturas complejas del radio distal constituyen un reto terapéutico muy importante, pues aparte de afectar gravemente las superficies articulares radiocarpiana y radiocubital distal, se les asocia una conminución y colapso metafisario que las hacen inherentemente inestables y, por tanto, difícilmente tratables por métodos conservadores ${ }^{1-3}$. En este sentido, la falta de reducción de la anatomía normal, tanto intra como extraarticular, tiene como consecuencia la aparición de dolor, la pérdida de movilidad y fuerza, y el desarrollo de una artrosis secundaria por la incongruencia articular residual ${ }^{4-9}$. Es por ello, que en la actualidad, se recomiende la fijación interna con placas y/o fijadores externos para tratar este tipo de lesiones ${ }^{7,10-12}$. En cuanto a la fijación interna con placas, existe una gran controversia en cuanto si es mejor colocarlas dorsalmente o en su vertiente palmar.

El objetivo de este estudio es dar a conocer los resultados radiológicos y funcionales de una serie de fracturas graves del extremo distal del radio (Tipo $\mathrm{C} 3$ de la clasificación de la $\mathrm{AO})^{13}$ tratadas con la placa dorsal «Pi», como único método de fijación interna.

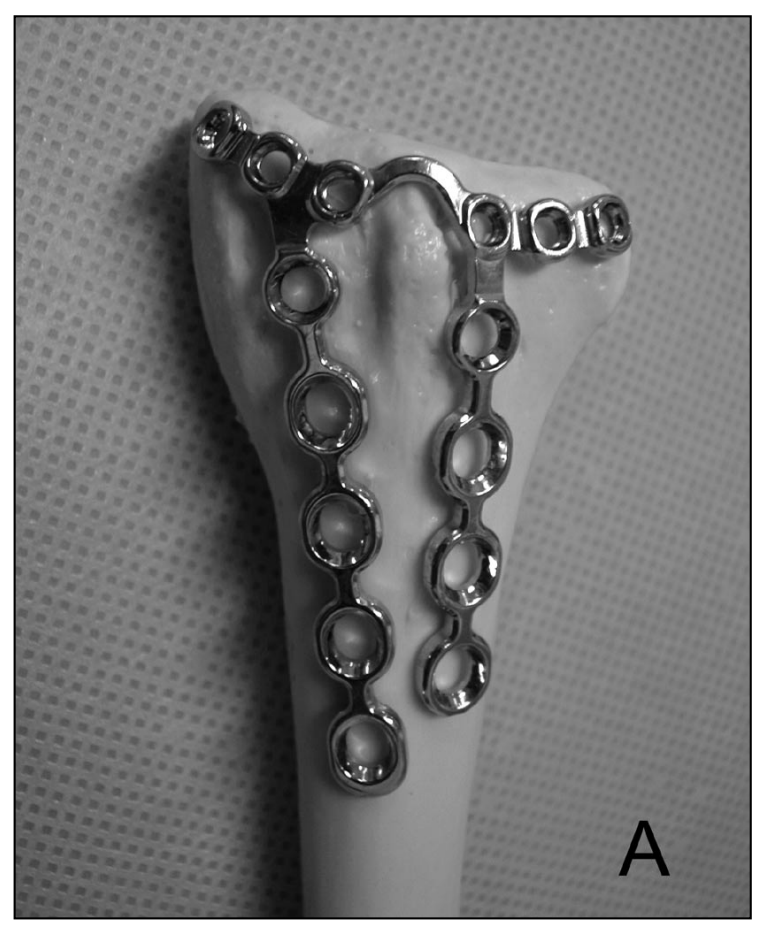

\section{MATERIAL Y MÉTODOS}

De enero de 2001 a enero de 2004, se trataron quirúrgicamente en nuestro servicio 243 pacientes con fracturas del extremo distal del radio. En 29 pacientes (12\%), con 30 fracturas (1 bilateral), se utilizó como material de osteosíntesis la placa Pi (Synthes®; Oberdof, Suiza) (Figura 1). De ellos hemos seleccionado para el estudio, 23 pacientes, con 24 fracturas (1 bilateral), que cumplían los siguientes criterios de inclusión: 1) fracturas tipo C3 (AO), 2) un mínimo de 6 meses de seguimiento y 3) fracturas con desplazamiento dorsal del radio distal.

Quince eran varones y 8 mujeres, con una media de edad de 53 años (rango 25/78 años). La muñeca derecha se lesionó en 10 pacientes y la izquierda en 14. La muñeca dominante fracturada coincidió en 8 pacientes (35\%). En cuanto a la etiología, 7 casos correspondieron a caídas casuales desde su altura, 6 por caídas desde una altura superior a un metro, 4 por accidentes de tráfico, 3 por accidentes deportivos y los otros 3, por una caída desde un caballo.

Siete pacientes $(30 \%)$ sufrieron lesiones asociadas, 3 de los cuales presentaron una fractura de la muñeca contralateral. La síntesis empleada para estas fracturas fue: la placa Pi en ambas muñecas en un paciente, mientras que en los otros dos, se utilizó la síntesis percutánea con agujas de Kirschner en la otra muñeca. Asimismo, de estos pacientes con fractura bilateral de ambas muñecas, dos sufrieron otras lesiones esqueléticas fuera de la extremidad

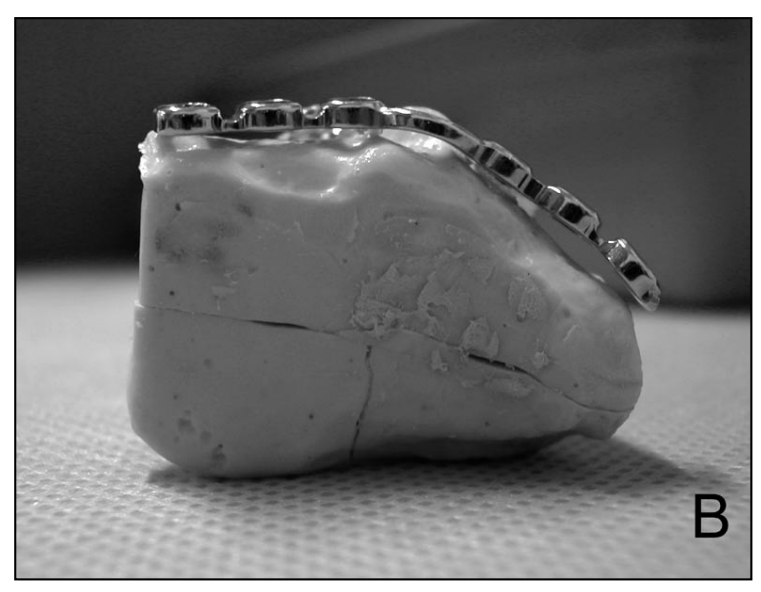

Figura 1. Placa «Pi» acoplada al extremo distal del radio. A) visión frontal. B) visión transversal. 

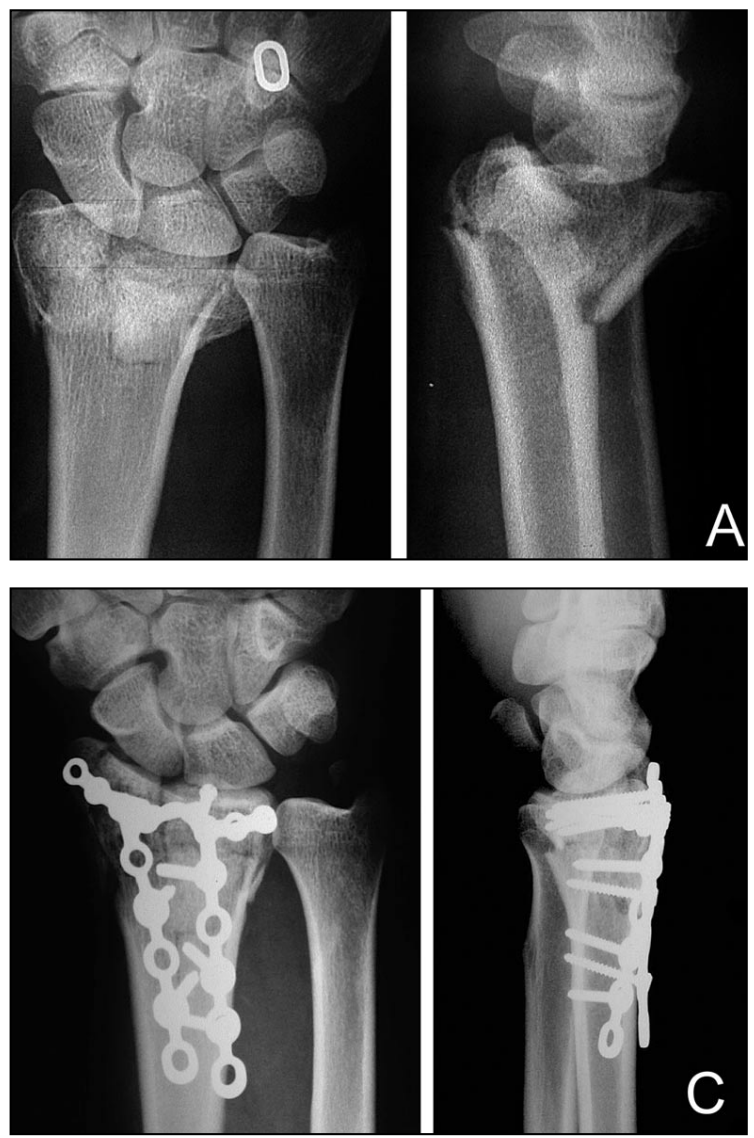

Figura 2. Tratamiento de una fractura del extremo distal del radio tipo C3. (A) Radiografías anteroposterior y perfil iniciales. (B) Tomografía axial computarizada donde se observa la conminución articular y el defecto óseo a nivel metafiasrio. (C) Reducción y osteosintesis con placa dorsal. Los tornillos y clavos distales, matienen la reducción articular.

afecta. De los 4 restantes, 2 sufrieron lesiones carpianas (una disociación escafolunar y una fractura de escafoides respectivamente), otro fractura del húmero homolateral, y el último, fractura del segundo metacarpiano homolateral, por la colocación incorrecta de los clavos del fijador externo con la que fue tratado inicialmente en otra institución.

Todas las fracturas se clasificaron según lo establecido por el gupo $\mathrm{AO}^{13}$. Así, de las fracturas tratadas en nuestra serie, $6(25 \%)$ correspondieron al tipo $\mathrm{C} 3.1,17(71 \%)$ al tipo $\mathrm{C} 3.2$ y $1(4 \%)$ al tipo C3.3.

En todos los casos se realizó un abordaje dorsal, con incisión longitudinal, utilizando el tubérculo de Lister como referencia anatómica y/ o el eje del tercer metacarpiano en caso de conminución del primero. Después de incidir lon-

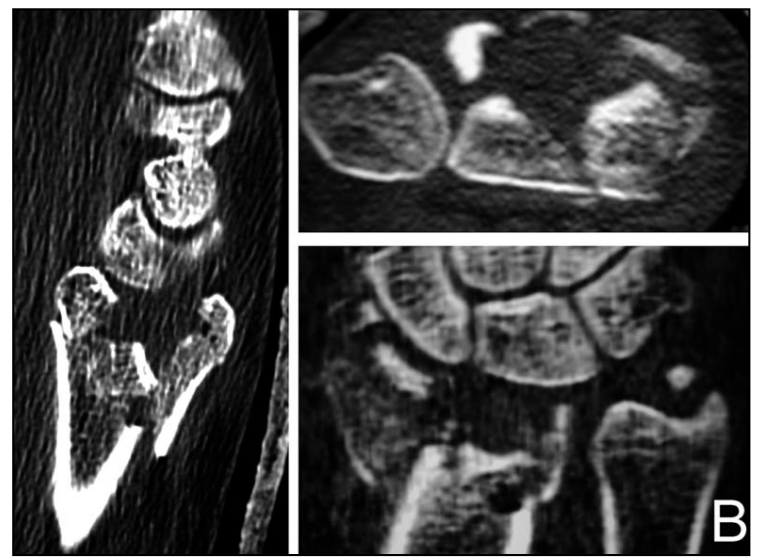

gitudinalmente el tercer compartimento, se aisló y rechazó radialmente el extensor largo del pulgar. Mediante disección subperióstica, se levantó el suelo retinacular del segundo y del cuarto compartimento logrando, de esta manera, una exposición adecuada del foco de fractura. En este último paso es imprescindible el uso de una técnica atraumática para respetar la indemnidad del suelo del compartimento, elemento anatómico fundamental para la cobertura de la placa. La reducción extraarticular se realizó mediante distracción manual, no siendo necesario el uso de fijador externo. En algunos casos se emplearon agujas de Kirschner para mantener temporalmente la reducción antes de la colocación de la placa. Los fragmentos articulares se redujeron a través de la conminución cortical metafisaria dorsal, utilizando instrumentos romos para su manipulación y reducción desde el hueso subcondral. Bajo visión radioscópica se evaluó la congruencia articular. Se acopló la placa a la diáfisis radial por medio de sus 2 ramas paralelas longitudinales, siendo fijadas al hueso con tornillos de $2.7 \mathrm{~mm}$, mientras que su rama transversa distal se solidarizaba a los fragmentos dorsales mediante tornillos de $2.4 \mathrm{~mm}$ y/o clavos de soporte de 1.8 mm (Figura 2). Finalmente, se cerró la herida por planos, teniendo la precaución de cubrir la placa con el periostio del segundo y cuarto compartimientos extensores. En los casos en que este no fuera suficiente para separar los tendones extensores de la rama transversa de la placa, se utilizó una bandeleta del techo del retináculo extensor, según la técnica descrita por Chiang et $a l .{ }^{14}$. El tendón extensor largo del pulgar se dejó en el plano subcutáneo. 


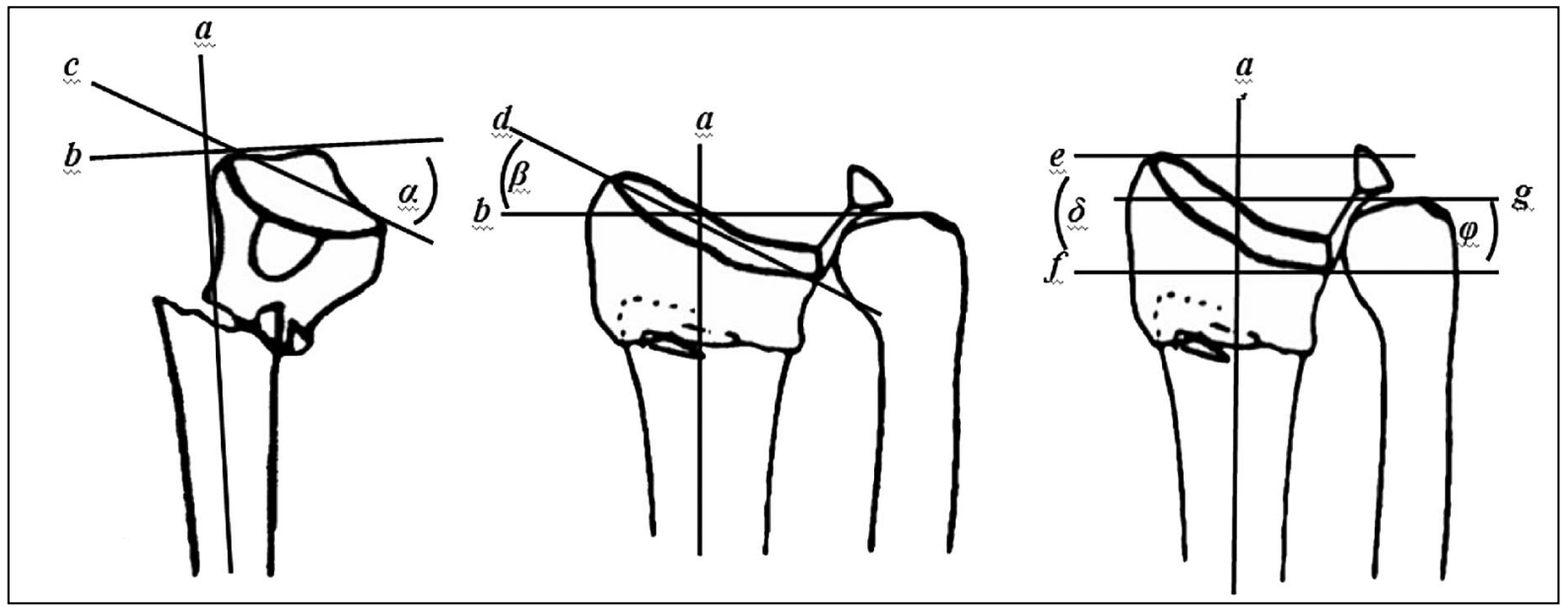

Figura 3. Relaciones anatómicas del extremo distal del radio. (a) Inclinación palmar. $(\beta)$ Inclinación Radial. $(\delta)$ Longitud Radial. ( $\varphi$ ) Varianza Cubital. (a) Eje longitudinal del radio. (b) Línea perpendicular a línea a. (c) Línea paralela a la superficie articular del radio de perfil. (d) Línea paralela a la superficie articular del radio de frente. (e) Línea perpendicular al eje del radio que pasa por el extremo distal del mismo. (f) Línea perpendicular al eje del radio que pasa por extremo distal cubital del radio. (g) Línea perpendicular al eje del radio que pasa por el extremo distal del cúbito..

Como medidas adicionales a la placa, se empleó injerto óseo en 8 fracturas ( 7 de olécranon y uno de cresta ilíaca). En uno de los pacientes en que se utilizó injerto óseo de olécranon se agregó, además, polimetilmetacrilato para rellenar la pérdida de hueso metafisario.

Todos los pacientes fueron inmovilizados con vendaje enyesado por debajo del codo por un período máximo de 4 semanas. Inmediatamente después de la cirugía se instó al paciente a movilizar activamente dedos, codo y hombro y, una vez retirada la inmovilización, se inició tratamiento rehabilitador supervisado por fisioterapeuta.

Al final del estudio, se midió con goniómetro el arco de movilidad de flexoextensión, desviaciones radial y cubital y pronosupinación, mientras que la fuerza de puño se objetivó con un dinamómetro Jamar digital, comparando los valores obtenidos con los del lado sano.

En cuanto a los parámetros radiográficos, se midió la altura radial, las inclinaciones palmar y radial, así como la varianza cubital pre y postquirúrgica (Figura 3). Aquí también, los resultados se compararon con el lado sano. Sin embargo, en los casos con fractura bilateral, se tomó como parámetros medios normales radiológicos los descritos por Szabo y Weber ${ }^{15}$ : in- clinación palmar $11^{\circ}$, inclinación radial $23^{\circ}$ y altura radial $12 \mathrm{~mm}$. La congruencia articular se evaluó intraoperatoriamente bajo visión radioscópica. Debido a que los escalones articulares se midieron en las radiografias pre y postoperatorias, la superposición de la rama transversa de la placa con la superficie articular imposibilitó esta medición en las radiografías postoperatorias de siete pacientes, en quienes el escalón articular se midió en la radiografía realizada después de la extracción de la osteosíntesis. En cuanto a los resultados globales, se utilizó la escala de Gartland y Werley ${ }^{16}$.

\section{RESULTADOS}

El seguimiento de los pacientes fue de 22 meses de media (rango 7-42 meses).

La corrección promedio que se obtuvo radiográficamente de los parámetros valorados fue: para la inclinación palmar, de $26^{\circ}$ dorsal (rango $\left.-50^{\circ} / 5^{\circ}\right)$ en el preoperatorio a $4^{\circ}$ palmar (rango $-20^{\circ} / 20^{\circ}$ ) en el postoperatorio; para la inclinación radial, de $18^{\circ}$ (rango $-8^{\circ} / 34^{\circ}$ ) en el preoperatorio a $22^{\circ}$ (rango $\left.16^{\circ} / 29^{\circ}\right)$ en el postoperatorio, y la longitud radial, de $10 \mathrm{~mm}$ (rango 0/16) en el preoperatorio a $11 \mathrm{~mm}$ (rango 
7/16) en el postoperatorio. La varianza cubital se corrigió de $+2 \mathrm{~mm}$ (rango $-10 /+10$ ) a $0 \mathrm{~mm}$ (rango -8/+7). Resumiendo dichos valores en porcentajes, observamos que la restitución de la inclinación palmar fue del $60 \%$, la inclinación radial del $81 \%$, y la longitud radial del $92 \%$. En los tres pacientes que presentaron ambas fracturas de muñeca, los resultados se compararon con los valores normales de la población adulta publicados por Szabo y Weber ${ }^{15}$. El escalón articular se corrigió de $2.5 \mathrm{~mm}$ (rango 0/11) a $0.5 \mathrm{~mm}$ (rango 0/4). En las radiografías a largo plazo de dos pacientes no se pudo medir el escalón articular por la superposición con la osteosíntesis. La reducción fue evaluada previamente en quirófano bajo visión radioscópica.

La valoración funcional de la movilidad se llevó a cabo en todos los pacientes. Sin embargo, para evitar sesgos en la misma, los pacientes que habían sufrido lesiones en la muñeca contralateral no se incluyeron en los resultados comparativos, por lo que solo se valoraron los resultados de la muñeca lesionada. Así, el rango de movilidad en los 20 pacientes con lesión unilateral fue de: $112^{\circ}$ promedio de flexo-extensión, $60^{\circ}$ promedio de desviaciones radial y cubital y $169^{\circ}$ promedio de pronosupinación.

La fuerza media de prensión fue de $35,8 \mathrm{Kg}$. (rango 4,5/68,5 Kg.) en el lado lesionado y de 41,2 Kg. (rango 13,2-77,6 Kg.) en el lado sano. En porcentaje, el promedio de recuperación fue del $87 \%$.

Se tuvo que retirar el implante en 9 muñecas $(40 \%)$, en un tiempo postoperatorio medio de 8 meses (rango 4/16 meses). Un caso fue debido a una tendinitis de flexores por un tornillo que protruía por la cortical volar del radio; en ocho pacientes por leves molestias referidas como sensación de cuerpo extraño, y en el otro caso por una tendinitis crepitante de extensores. No obstante, la extracción del implante fue seguida de una mejoría completa de la sintomatología, en todos los casos, sin presentar problemas con este segundo procedimiento quirúrgico.

Con respecto a las complicaciones, solo un caso presentó una distrofia simpática refleja, pero que evolucionó favorablemente después de la extracción del implante y rehabilitación asis- tida. No hubo que lamentar infecciones, pseudoartosis, compresión del nervio mediano, aflojamiento de tornillos, fallo del material de osteosíntesis ni rupturas tendinosas.

Finalmente y de acuerdo a la escala de Garland y Werley ${ }^{16}$, en el $67 \%$ de los casos se obtuvieron excelentes resultados y en el $33 \%$ restante, buenos. No hubo ningún caso de resultados regulares ni malos.

\section{DISCUSIÓN}

El objetivo en el tratamiento de las fracturas complejas del extremo distal del radio, es obtener un buen resultado funcional e indoloro, lo que está relacionado directamente con la restitución de la anatomía, incluyendo la congruencia articular, ángulos radiales, así como la longitud radial ${ }^{4-9}$. La clasificación del grupo AO (Arbeitsgemeinschaft für Osteosynthesenfragen) agrupa estas lesiones graves dentro del Tipo $\mathrm{C} 3{ }^{13}$. Es este mismo grupo $\mathrm{AO}$, quién diseñó la placa «Pi» de bajo perfil que se adapta a la anatomía dorsal del radio distal con el objetivo de facilitar su uso y limitar las complicaciones que aparecían con el empleo de otras placas ${ }^{17,18}$. Ring et $\mathrm{al}^{17}$, grupo responsable del desarrollo del implante, publicaron en 1997, los primeros resultados con la utilización de esta placa en una serie de 22 pacientes con fracturas tipos $\mathrm{C} 2$ y $\mathrm{C} 3$, pero sin discernir el número de casos para cada una de ellas. Se han publicado cuatro series más que valoran los resultados y complicaciones de pacientes tratados con este implante ${ }^{14,19-21}$, aunque el número de fracturas tipo $\mathrm{C} 3$, de un total de 85 fracturas, solo sumaron 32 casos. Sánchez et al recientemente publicaron las complicaciones en 389 pacientes con fracturas articulares tratadas con placa $\mathrm{Pi}^{22}$.

A excepción del trabajo de Hanhnloser et $\mathrm{al}^{20}$ en que contraindican el uso de la placa «Pi» para este tipo de fracturas, las demás series aportan unos resultados comparables a los nuestros, tanto en el aspecto clínico como radiográfico ${ }^{14}$, 17, 19. En este sentido, este implante presenta características biomecánicas que deben ser tenidas en cuenta para el tratamiento de estas lesiones y principalmente para disminuir las complica- 
ciones que derivan de su utilización incorrecta. Por un lado, la forma de la placa que remeda la letra griega $\pi$, presenta la ventaja de poder ser usada con efecto de sostén por la rama distal transversa que abraza toda la circunferencia dorsal del radio. Esta tiene, además, la posibilidad de ser colocada sobre el borde dorsal distal del radio reduciendo y manteniendo la conminución que habitualmente está asociada a las fracturas tipo C3. Asimismo, la fijación distal puede realizarse por medio de la fijación rígida que brindan los tornillos de $2.4 \mathrm{~mm}$. cuando el tamaño del fragmento lo permite o mediante los clavos bloqueados que introducen el concepto de estabilidad angular para el sostén subcondral de la conminución articular ${ }^{23}$, brindando la posibilidad de una reducción articular correcta y evitando el acortamiento radial. Estas últimas características hacen poco probable el aflojamiento distal de los tornillos o los clavos, por lo que consideramos que los fallos publicados por algunos autores, se deben principalmente a un error en la técnica, más que a un fallo del implante.

La principal complicación con la utilización de la placa «Pi», es la irritación de los tendones extensores, aunque también hay que considerar, como con cualquier placa dorsal, la posibilidad de pérdida de la reducción y/o el fallo del implante ${ }^{10,11}$. La primera descripción de rotura tendinosa asociada a la placa « $\mathrm{Pi} »$, fue publicada por Kambouroglou y Axelrod ${ }^{24}$ en 2 pacientes. Desde entonces, se han comunicado otros $\operatorname{casos}^{14,19,21}$, incluyendo la ruptura del fle- xor largo del pulgar por la utilización palmar de la placa $\mathrm{Pi}^{25}$. Con el fin de minimizar este problema, recomendamos la extracción del implante en los pacientes con dolor o crepitación tendinosa o en aquellos casos en los que no sea seguro un seguimiento periódico. Aunque este segundo acto pueda ser criticable, coincidimos con Sánchez ${ }^{22}$, en que no modifica el resultado. En este tipo de fracturas lo que prima en el resultado a largo plazo es conseguir una buena reducción anatómica ${ }^{8}$, y, en nuestra experiencia, la placa «Pi» consigue este objetivo.

En conclusión y de acuerdo con los resultados que presentamos en esta serie de pacientes, la osteosíntesis con placa «Pi» de las fracturas del extremo distal del radio tipo $\mathrm{C} 3$ de la $\mathrm{AO}$, constituye una alternativa válida en el tratamiento de estas lesiones, ya que las características biomecánicas del implante y la correcta utilización de éste, permiten: 1) tratar el desplazamiento dorsal con un efecto de sostén; 2) solventar la conminución metafisaria dorsal y el acortamiento radial, al mantener la longitud del radio por proporcionar fijación rígida distal y proximal; y 3) restablecer la congruencia articular radiocarpiana y radiocubital distal a través de la fijación fragmentaria y la estabilidad angular.

Sin embargo, creemos que para validar estos resultados, se necesitaría efectuar un estudio prospectivo, comparando aleatoriamente una serie de fracturas tratadas con placa «Pi» con otra serie de fracturas, del mismo tipo, pero tratadas con placa palmar. 


\section{BIBLIOGRAFÍA}

1. Hastings, II H.; Leibovic, S.: Indications and techniques of open reduction: internal fixation of distal radius fractures. Orthop Clin North Am, 1993; 24: 309-326.

2. Jupiter, J., Fernández, D., Whipple, T., et al.: Intra-articular fractures of the distal radius: Contemporary perspectives. AAOS Instr Course Lect, 1998; 47: 191-203.

3. Leibovic, S.; Geissler, W.: Treatment of complex intra-articular distal radius fractures. Orthop Clin North Am, 1994; 25: 685-706.

4. Fernández, D.: Should anatomic reduction be pursued in distal radial fractures? J. Hand Surg, 2000; 25B: 6: 523-527.

5. Fernández, D.; Geissler, W.: Treatment of displaced articular fractures the radius. J Hand Surg, 1991; 16A: 375-384.

6. Gliatis, J.; Plessas, J.; Davis, R.: Outcome of distal radial fractures in young adults. J. Hand Surg, 2000; 25B: 6:535-543.

7. Jupiter, J.; Lipton, H.: The operative treatment of intraarticular fractures of the distal radius. Clin Orthop, 1993, 292: 48-61.

8. Knirk, J.; Jupiter, J.: Intra-articular fractures of the distal end of the radius in young adults. J. Bone Joint Surg, 1986; 68A: 647-659.

9. Trumble, T.; Schmitt, S.; Vedder, N.: Factors affecting functional outcome of displaced intra-articular distal radius fractures. J. Hand Surg, 1994; 19A: 325340.

10. Fitoussi, F.; Ip, W.; Chow S.: Treatment of displaced intraarticular fractures of the distal end of the radius with plate. J. Bone Joint Surg, 1997; 79A: 1303-1312.

11. Ring, D.; Prommersberger, K.; Jupiter, J.: Combined dorsal and volar plate fixation of complex fractures of the distal part of the radius. J. Bone Joint Surg, 2004; 86A: 1646-1652.

12. Rogachefsky, R.; Lipson, S.; Applegate, B. et al.: Treatment of severely comminuted intraarticular fractures of the distal end of the radius by open reduction and combined internal and external fixation. J. Bone Joint Surg, 2001; 83A: 509519.

13. Perren, S.; Müeller, M.; Schenk, R., et al.: Aspectos básicos de la osteosíntesis. Müeller, M.; Allgöwer, M.; Schneider, R.; Willenegger, H. (Eds.): Manual de osteosíntesis. Sringer-Verlag Ibérica, 1993: 1-158.

14. Chiang, P.; Roach, S.; Baratz, M.: Failure of a retinacular flap to prevent dorsal wrist pain after titanium pi plate fixation of the distal radius fractures. J. Hand Surg, 2002; 27A: 724728.

15. Szabo, R.; Weber, S.: Comminuted intraarticular fractures of the distal radius. Clin Orthop, 1988; 230: 39-48.

16. Gartland, J.; Werley, C.: Evaluation of healed Colles' fractures. J. Bone Joint Surg, 1951, 33A: 895-907.

17. Ring, D.; Jupiter, J., Brennwald, J. et al.: Prospective multicenter trial of a plate for dorsal fixation of distal radius fractures. J Hand Surg, 1997; 22A: 777-784.

18. Peine, R.; Rikli, D.; Hoffmann, R. et al.: Comparison of three different plating techniques for the dorsum of the distal radius: A biomechanical study. J. Hand Surg, 2000; 25A: 29-33.

19. Campbell, D.: Open reduction and internal fixation of intra-articular and unstable fractures of the distal radius using the AO distal radius plate. J. Hand Surg, 2000; 25B: 6:528-534.

20. Hahnloser, D.; Platz, A.; Amgwerd, M. et al.: Internal fixation of distal radius fractures with dorsal dislocation: pi-plate or two? tube plates? A prospective randomized study. J. Trauma, 1999; 47: 760-765.

21. Rozental, T.; Beredjiklian, P.; Bozentka, D.: Functional outcome and complications following two types of dorsal plating for unstable fractures of the distal part of the radius. J. Bone Joint Surg, 2003; 85: 1956-1960.

22. Sánchez, T.; Jakubietz, M.; Jakubietz, R.; Mayer, J.; Beutel, F.; Grünert, J.: Complications after pi plate osteosynthesis. Plast Reconstr Surg 2005, 116: 153-158.

23. Wagner, M.: General principles for the clinical use of the LCP. Injury, 2003; 34, suppl. 2: 31-42.

24. Kambouroglou, G.; Axelrod, T.: Complications of the AO/ASIF titanium distal radius plate system ( $\pi$ plate) in internal fixation of the distal radius: $A$ brief report. J Hand Surg, 1998, 23A: 737-741.

25. Nunley, J., Rowan, P.: Delayed ruptured of the flexor pollicis longus tendon after inappropriate placement of the $\pi$ plate on the volar surface of the distal radius. J. Hand Surg, 1999; 24A: 1279-1280. 